\title{
Design for a Digital Image Processing Platform based on GUI
}

\author{
Dan Tian*, Yue Zheng and Dong-dong Yao \\ School of Information Engineering, Shenyang University, Shenyang, 110044, China
}

\begin{abstract}
Keywords: Digital image processing platform, Image denoising, Image segmentation, Image mosaic, GUI.

Abstract. A digital image processing experimental platform is designed based on MATLAB GUI. This platform has good interactivity and expansibility, which integrates multiple functional modules including low-level processing module, noise adding module, image denoising module, image segmentation module, and image mosaic module. The platform can help students learning the basic theory and methods for digital image processing.
\end{abstract}

\section{Introduction}

MATLAB GUI ${ }^{[1]}$ (Graphical User Interface) provides a visual interactive platform, which can be used for settling code uniformly. It is relatively easy to get familiar with and requires little knowledge of programming. This paper constructs a digital image processing experimental platform based on MATLAB GUI. The platform has five modules, which integrate low-level processing (rotation, brighten, graying, zooming, and restoration), noise adding (impulse noise, Gaussian noise, and multiple noise), image denoising ${ }^{[2]}$ (median filter and linear filter), image segmentation ${ }^{[3]}$ (sobel operator and fractional sobel operator), and image mosaic ${ }^{[4]}$ (corner detection ${ }^{[5]}$ and image mosaic) functions. We conduct the platform main interface based on M language. Clicking the arbitrarily submodule in the main interface, the corresponding submodule can be open and the corresponding function can be realized.

\section{Platform Construction}

MATLAB GUI is composed of the window, menu, icon, cursor, keys, dialog boxes, text and other graphical objects. It allows users to customize how to interact with MATLAB. This paper designs a digital image processing platform based on MATLAB GUI to realize the basic function in digital image processing. Default user interface is shown in Figure.1.

The menu bar is composed of file, image processing, and restoration menu. Click the file menu, we can add the original image $\mathrm{A}$ and $\mathrm{B}$, and save the image processing results. Click the image processing menu, we can select the corresponding image processing function shown in Figure.2. Click the restoration menu, the original image window and the processed image window can be restored. The toolbar offers the open, save and zooming functions.

Selecting the low-level processing module, we can realize the corresponding image processing function. The low-level processing module intergrades image rotation, image brighten, image graying, image zooming, and image restoration functions. Click the corresponding module, we can set the needed parameter. Such as, rotation angular, scaling plateau, etc. Figure. 3 shows the function of the image rotation module (rotation angular is set as $40^{\circ}$ ), which can conduct arbitrarily angular rotation for the image.

Selecting the noise module, we can add kinds of noise with specific intensity, such as Salt and pepper noise, Gaussian noise, and multiple noise. Salt and pepper noise is a kind of black and white bright dark spot noise. Gaussian noise is a kind of random noise, whose amplitude obeys Gaussian distribution. When the power spectral density of the Gaussian noise is uniformly distributed, it is named as Gaussian white noise. Multiple noise has the multiplication relationship with the image. Click the corresponding submodule, we can set the noise intensity and add noise. Figure. 4 shows the simulation result for adding Gaussian white noise, whose mean value is 0 , and variance is 0.02 . 


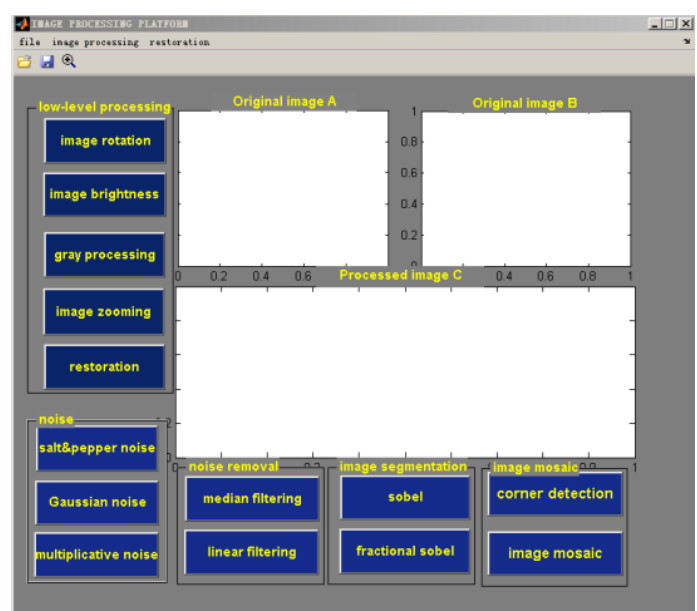

Figure 1. Default user interface

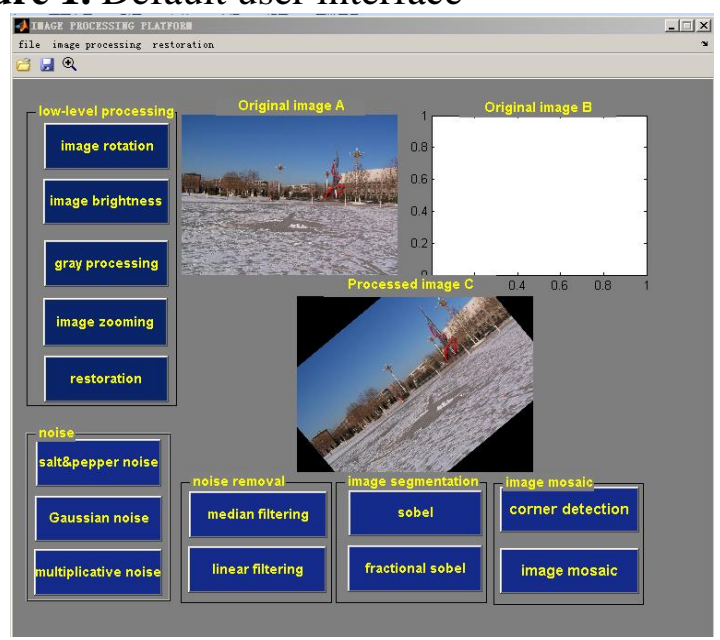

Figure 3. Image rotation module function

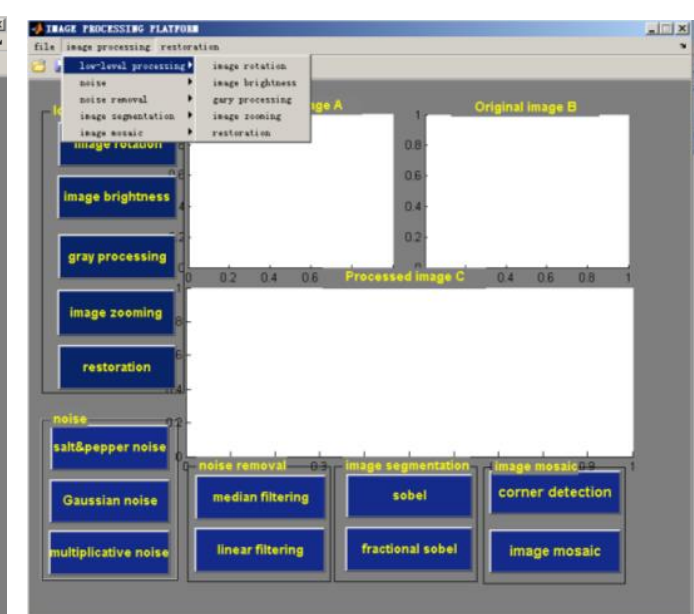

Figure 2. Image processing menu

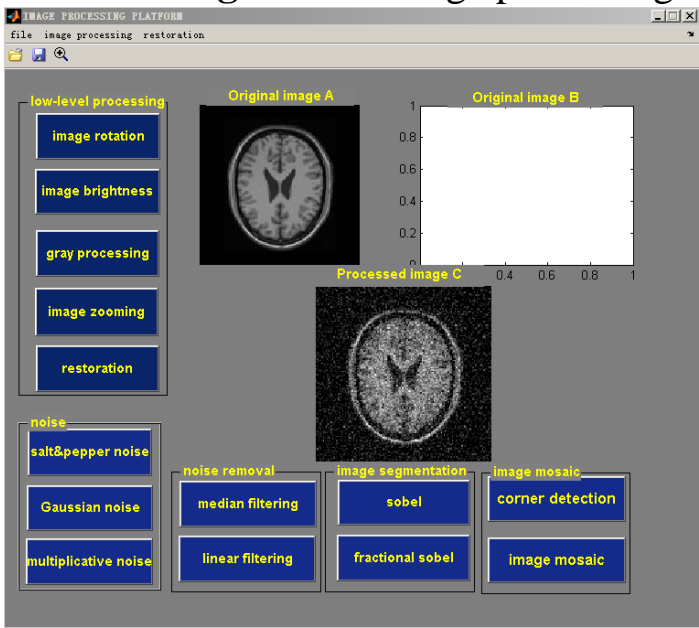

Figure 4. Noise adding module function

Selecting the noise removal module, we can process the image using median filter and linear filter methods respectively. Median filter is a kind of nonlinear smoothing technique. It sets the pixel gray value as the median of all pixels within a certain neighborhood window. Linear filter also processes the pixel gray directly, but by a linear smoothing way. Figure. 5 shows the simulation results using these two methods for denoising the noise image in Figure.4.

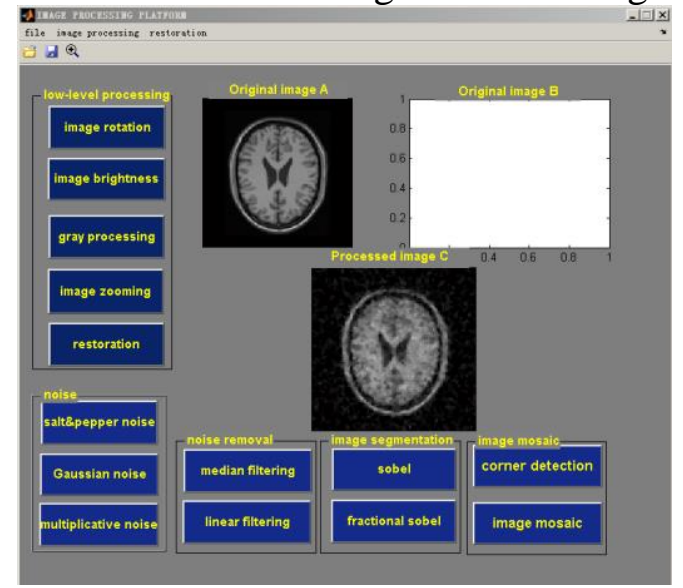

(a) median filter

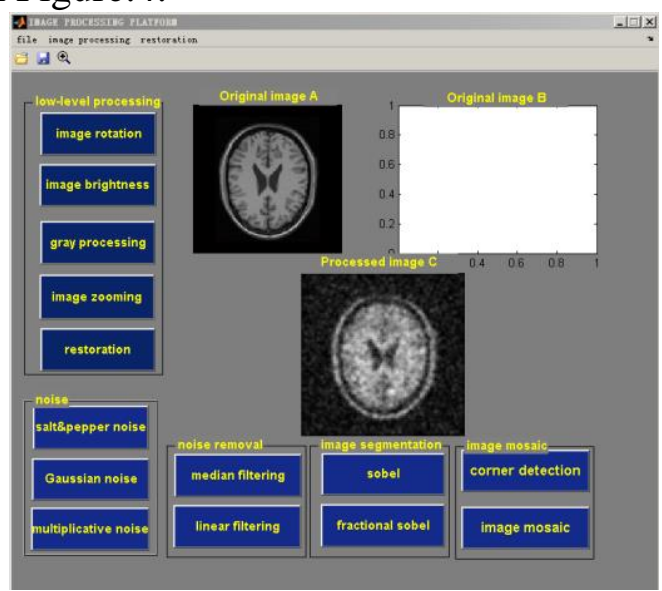

(b) linear filter

Figure5. Image denoising module function

Selecting the image segmentation module, we can segment the image using the sobel operator and fractional sobel ${ }^{[6]}$ operator respectively. Sobel is a classical gradient-based edge detection operator. The fractional sobel operator can be seen as generalization of the first-order Sobel operator. The goal is to utilize the frequency characteristic of the fractional derivative for extracting more structure feature details. Since the frequency characteristic determines it can preserve more low-frequency 
contour feature, keep high-frequency marginal feature and also enhance medium-frequency texture details. Figure. 6 shows the simulation results using these two methods.

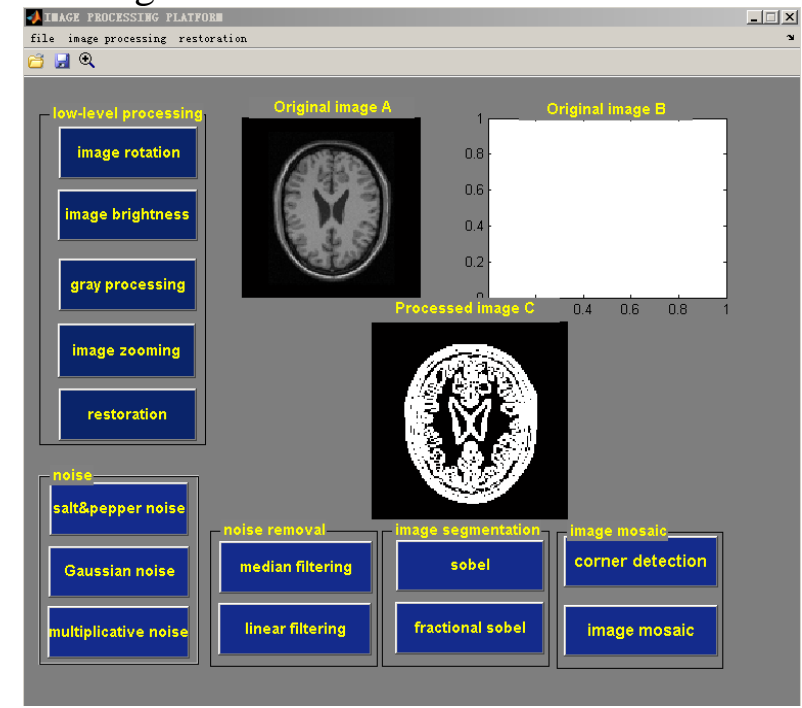

(a) sobel operator

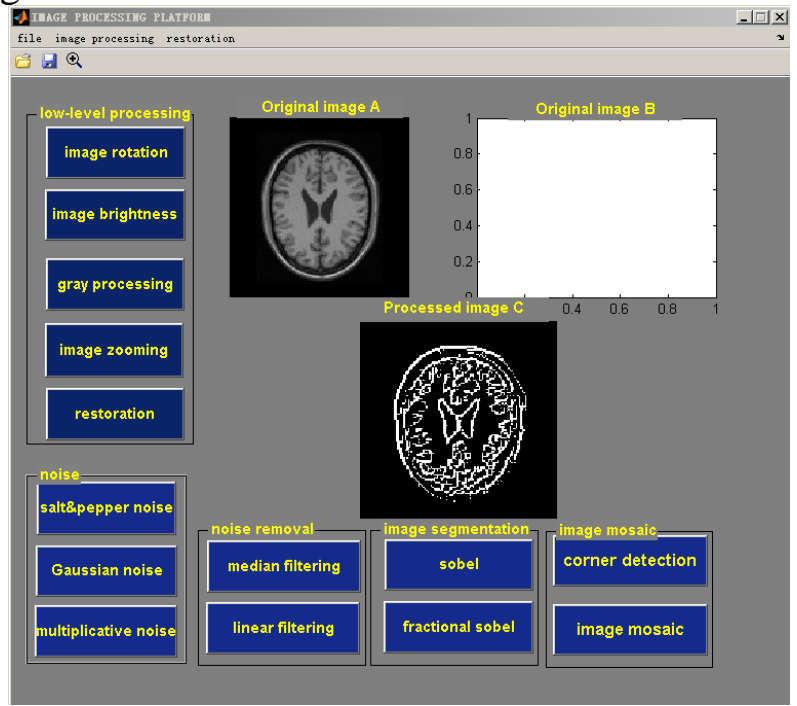

(b) fractional sobel operator

Figure 6. Image segmentation module function

Selecting the image mosaic module, we can realize the corner detection and image mosaic function respectively. Here the Harris corner detection method is used. The Harris is a kind of feature extraction operator, which applies the differential operation and auto correlation matrix operation to extract the corner. Image mosaic can joint two images having overlapping region. Figure. 7 shows the simulation result.

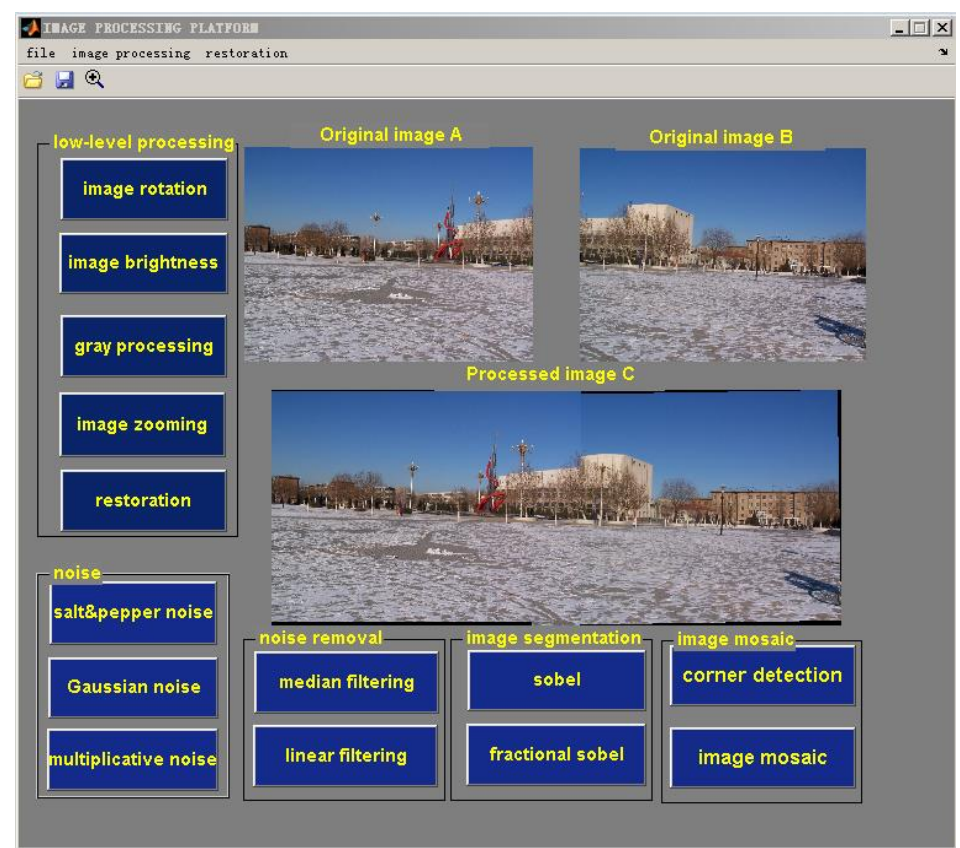

Figure7. Image mosaic module function

\section{Summary}

This paper presents the use of GUI tool for designing the digital image processing experimental platform based on MATLAB. MATLAB provides an easy way to build GUI with elements such as radio buttons, check boxes, pushbuttons, list boxes etc., and link them to bulid-in MATLAB functions. We utilize these controls to integrate some basic image processing functions, such as low-level processing, noise adding, noise removal, image segmentation, and image mosaic. The platform realizes good interactivity and expansibility. 


\section{Acknowledgement}

This research was financially supported by the Liaoning province Doctor Startup Foundation, China (Grant No. 201601213), and the Liaoning province College Students Innovation and Entrepreneurship Project, China (Grant No. 201611035000016).

\section{References}

[1]J.R. Raj, S.M.K. Rahman, S. Anand. Microcontroller USB interfacing with MATLAB GUI for low cost medical ultrasound scanners, Engineering Science and Technology, 2016, 19(2): 964-969.

[2]O. Tuzel, J. Thornton, J. Van Baar. Image denoising using a library of functions: U.S. Patent 9,262,810. (2016)

[3] P. Ghamisi, M.S. Couceiro, F.M.L. Martins, et al. Multilevel image segmentation based on fractional-order Darwinian particle swarm optimization. IEEE Transactions on Geoscience and Remote sensing, 2014, 52(5): 2382-2394.

[4] Q. Zhi, J.R. Cooperstock. Toward dynamic image mosaic generation with robustness to parallax. IEEE Transactions on Image Processing, 2012, 21(1): 366-378.1

[5] P. L. Shui, W.C. Zhang. Corner detection and classification using anisotropic directional derivative representations. IEEE Transactions on Image Processing, 2013, 22(8): 3204-3218.

[6] D. Tian , J.F. Wu, Y.J. Yang. A fractional-order Sobel operator for medical image structure feature extraction. Advanced Materials Research. Trans Tech Publications, 2014, 860: 2910-2913. 